\title{
ArcheoSciences
}

Revue d'archéométrie

\section{Le pigment d'aérinite dans deux peintures murales romanes du Sud-Ouest de la France}

Aerinite as a pigment found in two romanesque mural paintings in the SouthWest of France

Floréal Daniel, Barbara Laborde, Aurélie Mounier et Émilie Coulon

\section{(2) OpenEdition}

Journals

\section{Édition électronique}

URL : https://journals.openedition.org/archeosciences/987

DOI : 10.4000/archeosciences.987

ISBN : 978-2-7535-1597-0

ISSN : 2104-3728

Éditeur

Presses universitaires de Rennes

\section{Édition imprimée}

Date de publication : 31 décembre 2008

Pagination : 83-91

ISBN : 978-2-7535-0868-2

ISSN : $1960-1360$

\section{Référence électronique}

Floréal Daniel, Barbara Laborde, Aurélie Mounier et Émilie Coulon, « Le pigment d'aérinite dans deux peintures murales romanes du Sud-Ouest de la France », ArcheoSciences [En ligne], 32 | 2008, mis en ligne le 31 décembre 2011, consulté le 31 janvier 2022. URL : http://journals.openedition.org/ archeosciences/987 ; DOI : https://doi.org/10.4000/archeosciences.987 


\title{
Le pigment d'aérinite dans deux peintures murales romanes du Sud-Ouest de la France
}

\author{
Aerinite as a pigment found in two romanesque mural paintings \\ in the South-West of France
}

\author{
Floréal DANIEL*, Barbara LABORDE, \\ Aurélie Mounier et Émilie Coulon
}

\begin{abstract}
Résumé : L'inventaire des peintures murales où le pigment d'aérinite a été employé, correspond chronologiquement à la période de l'art roman et géographiquement, jusqu'ici, à une zone, au sud des Pyrénées, qui coïncide avec les gisements locaux de ce minéral argileux en Catalogne et en Aragon. La découverte en France, pour la première fois, de ce pigment dans les peintures de la chapelle de l'ancien logis abbatial de Moissac (XII ${ }^{e}$ siècle, Tarn-et-Garonne) et dans celles de l'église Saint-Nicolas de Nogaro (fin XI ${ }^{e}$ siècle, Gers), ainsi que les relations iconographiques et stylistiques de ces sites avec les sites espagnols, sont des indices de la circulation des ateliers et/ou des matières premières entre le nord de l'Espagne et le sud de la France à l'époque médiévale.

De plus, ce pigment apparaît sous des nuances variant du bleu profond au vert, comme c'est le cas à Saint-Nicolas de Nogaro, ce qui entraîne souvent une confusion avec l'emploi de terres vertes. Plusieurs facteurs peuvent expliquer ces différences de ton : l'origine du minéral, la granulométrie, l'état d'oxydation du fer constitutif de cette argile, l'état de déshydratation...

Nous avons d'une part, comparé et analysé par différentes méthodes physico-chimiques des échantillons issus de gisements en France, Espagne et Maroc. Cette caractérisation, notamment par micro-spectrométrie Raman, a servi de référence pour l'identification de ce pigment dans les deux peintures murales médiévales françaises. D’autre part, des mesures de couleur après chauffage de l'aérinite en atmosphère oxydante ont mis en évidence que le processus de déshydratation entraîne les variations de couleur du minéral. Il s'avère que la couleur verte ou " verdâtre » de l'aérinite peut être soit d'origine naturelle (et dépendant du gisement), soit provoquée par la chauffe volontaire du minéral à $400{ }^{\circ} \mathrm{C}$.
\end{abstract}

\begin{abstract}
The inventory of the mural paintings where the pigment of aerinite was employed, corresponds chronologically to the period of the Romanesque art, and geographically, to a zone of the south of the Pyrenees, which coincides with the local layers of this argillaceous mineral in Catalonia and Aragon. The discovery in France, for the first time, of this pigment in paintings of the vault of the old abbey home of Moissac (12 $2^{\text {th }}$ Century, Tarn-et-Garonne, France), and in those of the church Saint-Nicolas of Nogaro (end $11^{\text {th }}$ Century, Gers, France), as well as the iconographic relations and stylistics of these sites with the Spanish sites, are strong indices of the circulation of the workshops andlor raw materials between the north of Spain and the south of France at the medieval time.

Moreover, this pigment appears under nuances varying from major blue to green, as in the case of Saint-Nicolas of Nogaro, which often leads to a confusion with the use of green earths. Several factors can explain these differences in hue: the origin of mineral, the granulometry, the state of oxidation of the constitutive iron of this clay, the state of dehydration...

We, on the one hand, compared and analyzed by various physicochemical methods the samples resulting from geological layers in France, Spain and Morocco. This characterization, in particular by Raman micro-spectrometry, was used as reference for the identification of this pigment in the two French medieval mural paintings. In addition, measurements of color after heating the aerinite in oxidizing atmosphere highlighted that the process of dehydration involves the variations of color of this mineral. It proves that the green or greenish color of the aerinite can be either of natural origin (and depend on the layer), or caused by the voluntary heating of the mineral to $400^{\circ} \mathrm{C}$.
\end{abstract}

Mots clés : Pigment, Aérinite, Peintures murales, MEB/EDS, Spectrométrie Raman.

Keywords: Pigment, Aerinite, Mural painting, SEM/EDS, Raman spectrometry.

* Institut de Recherche sur les Archéomatériaux (IRAMAT, UMR 5060) - Université Bordeaux, CNRS. (fdaniel@u-bordeaux3.fr) 


\section{INTRODUCTION}

L'aérinite est découverte en 1876, par le minéralogiste allemand A. Von Lassaulx alors qu'il étudiait un échantillon provenant de la province d'Huesca (Aragon, Espagne). Il le baptise "aérinite" (du grec : aérinos) en référence à sa couleur bleu ciel.

Il s'agit d'un silicate d'aluminium et de calcium hydraté (d'altération ophique des zones triasiques), dont la formule chimique proposée dans la base de données RRUFF ${ }^{1}$ est la suivante : $\left(\mathrm{Ca}_{5.1} \mathrm{Na}_{0.5}\right)\left(\mathrm{Fe}^{3+}, \mathrm{Al}, \mathrm{Fe}^{2+}, \mathrm{Mg}\right)(\mathrm{Al}, \mathrm{Mg})_{6}\left[\mathrm{HSi}_{12} \mathrm{O}_{36}(\mathrm{O}\right.$ $\left.\mathrm{H})_{12}\right]\left[\left(\mathrm{CO}_{3}\right)_{1.2}\left(\mathrm{H}_{2} \mathrm{O}\right)_{12}\right]$.

Le plus grand nombre des gisements se trouve dans des zones proches des Pyrénées espagnoles (Porta et al., 1990) : en Aragon, dans la province de Huesca (Estropiñan del Castillo, Caserras, Juseu, Nacha), en Catalogne, dans la province de Lérida (Tartareu, Villanueva de Avellanes, Ager, Artesa de Segre, Hostalets de Tosts), en Navarre, en Andalousie, dans la province de Malaga (à Antequera). En France, un seul gisement a été découvert à Saint-Pandelon, près de Dax, dans le département des Landes. Par ailleurs, on en trouve aussi hors des Pyrénées, où d'autres gisements ont été découverts : au Maroc, dans le Moyen-Atlas et dans l'Anti-Atlas, à la périphérie de la ville de Ouarzazate; au nord de l'Italie; au Portugal (à Serra de Argo); aux ÉtatsUnis (Arizona).

Dans le nord de l'Espagne (Catalogne, Aragon, Principauté d'Andorre), ce minéral a été identifié et reconnu pour la première fois en 1990 dans plusieurs dizaines de peintures murales romanes dont, notamment, pour ne citer que les plus connues, celles de Santa-Maria de Taüll (Catalogne, $\mathrm{XII}^{\mathrm{e}}$ siècle) ou de Santa-Maria de Sigena (Aragon, $\mathrm{XII}^{\mathrm{e}}$ siècle) conservées au Musée National d'Art Catalan (Porta $e t$ al., 1990). L'inventaire des peintures murales où le pigment d'aérinite a été employé, correspond jusqu'ici, chronologiquement à la période de l'art roman et géographiquement, à une zone au sud des Pyrénées, qui coïncide avec les gisements locaux de ce minéral argileux en Catalogne et en Aragon. Bien qu'une étude réalisée au Laboratoire de Minéralogie de l'Université de Toulouse (UMR 5563), non publiée, a montré que l'aérinite peut également prendre des nuances de vert (El Aghchmi, 1981), il est remarquable que la bibliographie concernant l'aérinite se limite à son étude en tant que pigment bleu (Palet Casas et de Andres Llopis, 1992).

La partie méthodologique de ce travail a pour objectif, d'une part, de montrer expérimentalement, à partir d'échantillons géologiques d'aérinite que différentes couleurs peu-

1. Site web du projet $\operatorname{RRUFF}^{\mathrm{TM}}$ : [www.rruff.info]. vent être obtenues à partir du même matériau et d'autre part d'établir le spectre Raman de l'aérinite. De nombreuses méthodes permettent l'identification de ce minéral cependant la spectrométrie Raman est, depuis plus de 20 ans, une méthode rapide (Coupry, 1991) et adaptée à l'étude de micro-prélèvements et peut être utilisée in situ avec des équipements portables. Dans un deuxième temps, grâce à ces spectres Raman de référence, il s'agissait de montrer que l'utilisation de ce pigment permettant des tons verts aussi bien que bleus, a débordé les limites du nord de l'Espagne et qu'on en trouve des exemples dans le sud-ouest de la France. En effet, la proximité stylistique des peintures de l'ancien logis abbatial de Moissac avec celles de l'art roman catalan pouvait nous laisser supposer la circulation de pigments spécifiques de la peinture murale des Pyrénées et en particulier l'aérinite qui présente des nuances de bleu-vert caractéristiques.

\section{Présentation des sites et du matériel}

A l'occasion de récentes interventions de conservation et restauration des peintures murales de l'ancien logis abbatial de Moissac (Tarn et Garonne, XII ${ }^{\mathrm{e}}$ siècle) et de l'église SaintNicolas de Nogaro (Gers, $\mathrm{XI}^{\mathrm{e}}$ siècle), des prélèvements ont été effectués pour identifier les matériaux constitutifs (pigments, liants) et les techniques picturales employées.

Comparées à d'autres peintures murales de la même époque, dont les couleurs (blanc, noir, rouge, jaune) sont limitées à celles qu'il est possible d'obtenir à partir de matériaux locaux, les peintures de Moissac et de Nogaro sont dotées d'une large palette de couleurs et, en particulier, des variations de tonalité de verts et de bleus et de pièces de décor rapportées (dorures). La richesse de la décoration tient au statut du lieu dans lequel elles s'inscrivent (chapelle du logis abbatial de Moissac) et la nature des pigments, en particulier pour les bleus, dépend de la valeur symbolique de l'élément de décor.

Les peintures de l'ancien logis des abbés de Moissac (aujourd'hui Musée Marguerite Vidal des Arts et des Traditions Populaires) ont été étudiées et décrites récemment (Czerniak, 2003). Il s'agit de l'un des ultimes vestiges des bâtiments conventuels romans de la grande abbaye de Moissac. Cet édifice fut construit à la fin du XI siècle et remodelé plusieurs fois jusqu'à nos jours. Les peintures murales (datées entre 1188 et 1196) représentent un arbre de Jessé qui prenait naissance sur le mur occidental de l'oratoire et se développe sur la voûte de cette chapelle rectangulaire dont le fond est entièrement bleu et où plusieurs thèmes sont représentés : un collège apostolique suivi de 
prophètes tenant des phylactères, assis dans des lacis végétaux qui régissent l'organisation des décors (Photo 1), un christ Pantocrator (entouré des quatre symboles évangélistes) est assis dans une mandorle au niveau des apôtres tandis qu'une Vierge en Majesté est entourée des prophètes.

L'église Saint-Nicolas de Nogaro, datée de la moitié du $\mathrm{XI}^{\mathrm{e}}$ siècle, est orientée d'est en ouest sur le plan basilical et est composée d'une nef et de deux bas-côtés. Le chevet est formé d'une abside et deux absidioles en hémicycle. Les peintures murales se situent dans les absidioles nord et sud.

Dans l'absidiole nord, les peintures murales, également du XI ${ }^{\mathrm{e}}$ siècle, représentent la vie de saint Laurent. La palette est très colorée (jaune, rouge, brun, rose, noir, blanc, bleu et vert). Dans l'absidiole sud, les peintures représentent un Christ en majesté dans une mandorle, entouré du tétramorphe (symboles des quatre évangélistes) et d'anges. La palette est également variée avec du bleu intense et du vert.

Prélèvements : En collaboration avec les restaurateurs ${ }^{2}$, des micro-prélèvements $(<1 \mathrm{~mm})$ ont été réalisés dans les peintures de Moissac et dans les absidioles nord et sud de l'église de Nogaro. Nous ne présenterons ici que les bleus et les verts (Tableau 1) : trois de couleur bleu ciel pour les peintures de Moissac, deux échantillons verts et deux bleus pour celles de Nogaro.

Échantillons d'aérinite géologique de référence: L'échantillonnage utilisé pour l'étude des variations de couleur de l'aérinite en fonction du chauffage et pour établir des spectres Raman de référence est constitué de minéraux provenant de Saint-Pandelon (10359) dont le site a été identifié et décrit (Azambre et Monchoux, 1988), du Maroc (10360) dans une région du sud de l'Atlas, près de Ouarzazate et

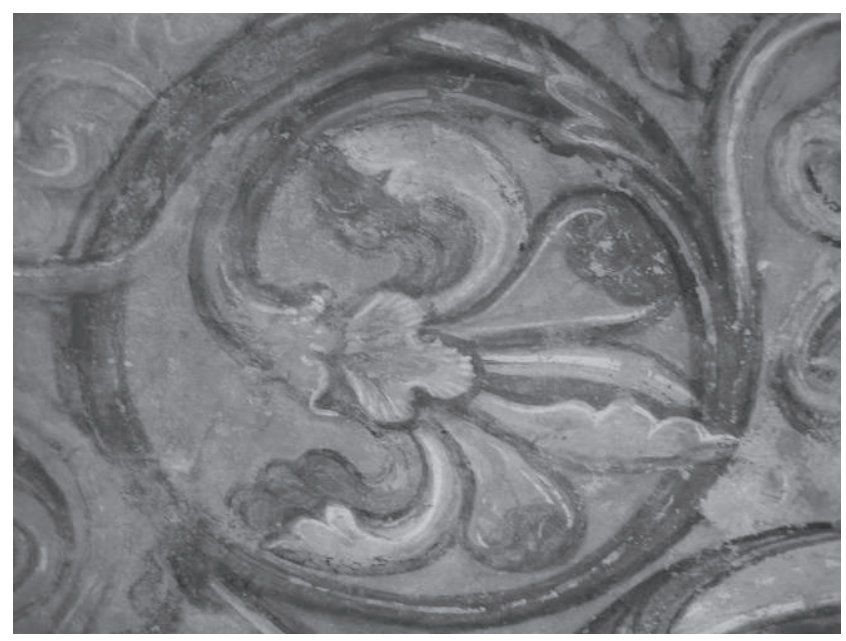

Photo 1 : Détail du décor floral (octopus flower) sur la voûte bleue de la chapelle de l'ancien logis abbatial de Moissac. (cliché CRP2A).

Picture 1: Detail of the floral decoration (octopus flower) on the blue vault of the chapel of the ancient abbey home in Moissac (Pict. CRP2A).

d'Espagne (10361) ${ }^{3}$ (entre la région d'Aragon et celle de Catalogne). Nous nous sommes assurés de la nature de ces échantillons en comparant les données fournies par la littérature à celle de nos analyses préalables (imagerie et analyse élémentaire par spectrométrie X couplé au microscope électronique à balayage [MEB/EDS] et analyse cristallographique par diffraction de rayons $\mathrm{X}[\mathrm{XRD}]$ ).

\begin{tabular}{|l|l|l|l|}
\hline & Réf. éch. & Localisation & Couleur \\
\hline \multirow{4}{*}{ Ancien logis abbatial de Moissac } & 10073 & Près de la tête du lion & bleu clair \\
\cline { 2 - 4 } & 10083 & Fond, derrière les volutes & bleu clair \\
\cline { 2 - 4 } & 10084 & Fond, derrière le visage d'un prophète & bleu clair \\
\hline \multirow{5}{*}{ Saint-Nicolas de Nogaro } & 10365 & Fond, absidiole nord & vert \\
\cline { 2 - 4 } & 10453 & Absidiole sud, près du bœuf (saint Luc) & vert \\
\cline { 2 - 4 } & 10457 & Sur la mandorle du christ & bleu \\
\cline { 2 - 4 } & 10460 & A droite de la tête du christ & bleu \\
\hline
\end{tabular}

Tableau 1 - Echantillons bleus et verts prélevés sur les peintures de Moissac et de Nogaro.

Table 1 - Blue and green samples from mural paintings of Moissac and Nogaro.

2. Jean-Marc Stouffs à Moissac et Didier Legrand à Nogaro.
3. Les échantillons d'aérinite d'Espagne et du Maroc ont été achetés dans le commerce. L'aérinite du gisement français nous a été aimablement fourni par les carrières Merle et Peyroux à Saint-Pandelon. 


\section{MÉTHOdes}

\section{Étude des variations de couleur de l'aérinite en fonction de sa déshydratation par chauffage et analyse par spectrométrie Raman}

On sait que les différentes nuances de couleur de l'aérinite peuvent êtres dues à l'état d'oxydation du fer et en particulier du rapport $\mathrm{Fe}^{2+} / \mathrm{Fe}^{3+}$ (El Aghchmi, 1981). Un chauffage en atmosphère oxydante permet de montrer ces variations de couleur. Pour provoquer le phénomène de déshydratation de l'aérinite, nous avons placé, dans un four "Carbolite " FC1600 programmable, deux des échantillons de référence (la quantité disponible d'aérinite de Saint-Pandelon était insuffisante), préalablement broyés dans un broyeur à billes (Retsch $S$ 100). Nous avons choisi de mesurer les variations de couleur à différentes températures : $300^{\circ} \mathrm{C}, 400{ }^{\circ} \mathrm{C}$, $600{ }^{\circ} \mathrm{C}$ et $1000^{\circ} \mathrm{C}$ avec une montée et une descente en température de $100^{\circ} \mathrm{C} /$ heure. Lorsque la température est atteinte, les échantillons sont laissés à la température voulue pendant 2 heures.

Les variations de la couleur ont été mesurées à l'aide d'un chromamètre portable (Minolta CR-100) donnant les coordonnées chromatiques, notamment, dans le système CIEL*a*b*.

Des images de la texture de l'aérinite ont été réalisées à l'aide d'un microscope électronique à balayage à pression variable (MEB, Jeol JSM-6460LV). Pour nous rapprocher des conditions expérimentales utilisées pour l'étude des prélèvements de peinture, nous avons travaillé en pression partielle $(15 \mathrm{~Pa})$ ce qui permet d'éviter la métallisation des échantillons et de pouvoir les réutiliser pour des analyses ultérieures. Ce mode ne permet d'obtenir des images qu'en électrons rétrodiffusés (BSE), toutefois le détecteur permet d'ajouter au contraste chimique propre aux images BSE une information topographique (mode shadow).

Le diffractogramme $\mathrm{X}$ de poudre de l'aérinite a été obtenu avant et après chauffage grâce à un appareil Siemens D500 équipé d'une anticathode de cuivre et un goniomètre Siemens type F. Les conditions opératoires sont les suivantes : anticathode $\mathrm{Cu} \mathrm{K} \alpha: \lambda=1,540 \AA$, filtre $\mathrm{Ni}$, tension : $40 \mathrm{kV}$, intensité : $30 \mathrm{~mA}$, acquisition de 15 à $50^{\circ} 2 \Theta$ avec un pas de $0,02 \%$. Ces angles ont été choisis en fonction de l'objectif qui était l'observer, après chauffage à différentes températures, la disparition des raies de l'aérinite et l'apparition de nouvelles phases d'oxydes de fer permettant d'expliquer les couleurs obtenues.

Les spectres Raman ont été obtenus grâce à un appareil Renishaw RM2000. Une caméra CCD permet la visualisation de l'échantillon en lumière naturelle sur un écran d'ordinateur et le logiciel informatique GRAMS permet de traiter les spectres. Les spectres obtenus ont été comparés à ceux de bases de données publiées (Bell et al., 1997). Lorsque les données faisaient défaut, comme c'était le cas pour l'aérinite, les spectres de référence ont été établis sur les échantillons géologiques. Notons que désormais un spectre Raman de l'aérinite est disponible dans la base de données RRUFF ${ }^{\mathrm{TM}}$ (Downs, 2006) au numéro de référence : R050610, établi pour un échantillon provenant d'Espagne (Estropiñan, province de Huesca, Aragon). Nous vérifions ici que cette référence est valable pour des échantillons d'autres provenances. Nos conditions opératoires sont les suivantes : Grandissement : x 50 ; puissance : $50 \mathrm{~W}$; type de laser : rouge- $633 \mathrm{~nm}(\mathrm{He}-\mathrm{Ne})$.

\section{Étude de prélèvements bleus et verts dans les peintures murales romanes de l'ancien logis abbatial de Moissac et de l'église Saint-Nicolas de Nogaro}

Lidentification des pigments est réalisée essentiellement par spectrométrie Raman. Les conditions expérimentales sont celles indiquées précédemment.

\section{Résultats ET DISCUSSION}

\section{Caractérisation de l'aérinite géologique}

Les résultats obtenus grâce à l'analyse des échantillons géologiques ont permis de confirmer la nature de ce minéral. Lobservation au microscope électronique à balayage (MEB) montre la structure fibreuse caractéristique des inosilicates (Figure 1). Les diffractogrammes $\mathrm{X}$ montrent des raies caractéristiques de l'aérinite (Azambre et Monchoux, 1988).

Les trois échantillons de référence ont permis d'obtenir des spectres Raman de l'aérinite. Le spectre de l'aérinite de Saint-Pandelon (10359) présente une bande d'intensité forte et large à $355 \mathrm{~cm}^{-1}$, une bande moyenne vers $525 \mathrm{~cm}^{-1}$ et des bandes plus faibles à 225 et $935 \mathrm{~cm}^{-1}$ (Figure 2). Ce spectre correspond à celui de la base de données RRUFF mentionnée plus haut. Une bande supplémentaire fine et intense apparaît dans le spectre Raman de l'échantillon du Maroc (10360) à $280 \mathrm{~cm}^{-1}$ qu peut être attribuée à la calcite et qui existe également sous la forme d'un épaulement dans le spectre Raman de l'échantillon provenant d'Espagne (10361). Celui-ci présente, de plus, un dédoublement de la bande principale à 335 et $360 \mathrm{~cm}^{-1}$. 


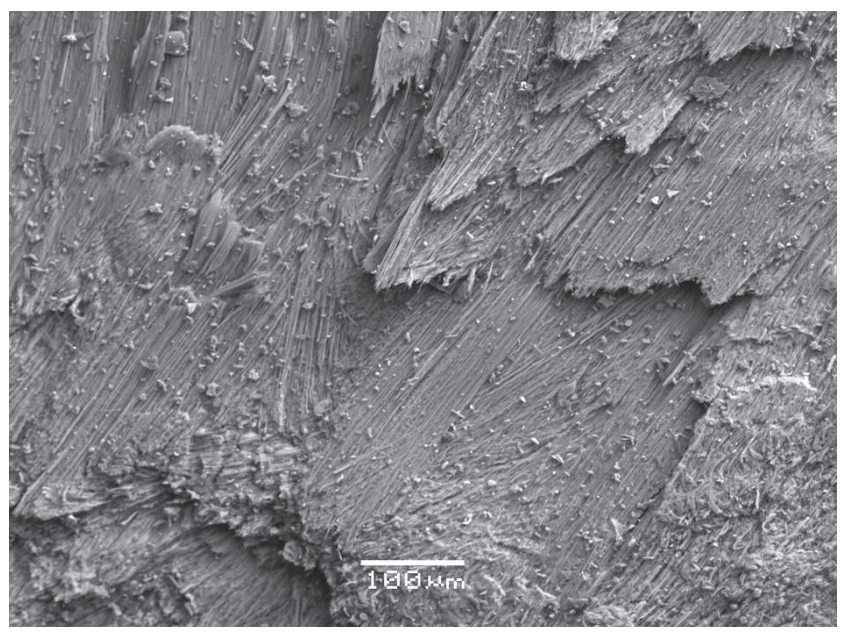

Figure 1: Image au microscope électronique à balayage en mode shadow (BST : électrons rétrodiffusés + topographie) de l'aérinite de Saint-Pandelon (France).

Figure 1: Image by scanning electron microscopy (shadow mode: back scattering electrons + topography) of aerinite from Saint-Pandelon (France).

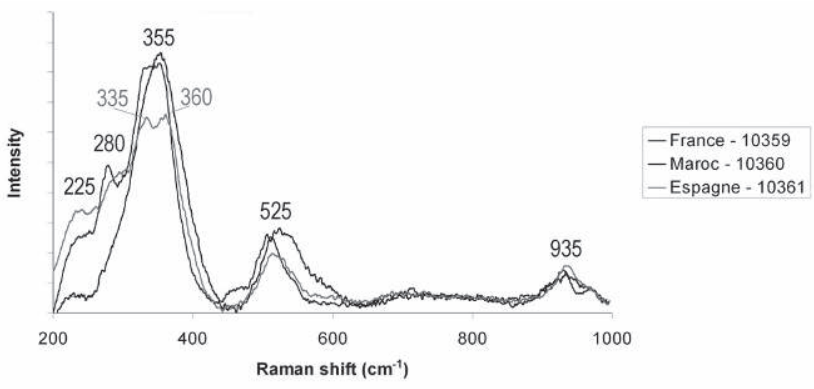

Figure 2 : Spectre Raman de l'aérinite de St-Pandelon, France (10359), du Maroc (10360) et d'Espagne (10361).

Figure 2: Raman spectra of aerinite from St Pandelon, France (10359), from Morocco (10360) and from Spain (10361).

\section{Effet du chauffage sur la couleur de l'aérinite}

Les études chromatiques et thermiques ont été menées simultanément sur les échantillons espagnols et marocains. Elles ont permis de mettre en évidence les modifications chromatiques subies par l'aérinite lors de l'augmentation de la température. La cuisson au four a permis une déshydratation entraînant la décoloration.

Ce n'est pas la perte de l'eau d'hygroscopicité liée à l'humidité de l'air, mais l'eau de cristallisation (ou de constitution) qui entraine des modifications au niveau des coordonnées chromatiques (El Aghchmi, 1981). Notons que nos échantillons espagnol et marocain n'ont pas à l'origine la même couleur. La valeur de $b^{*}$ est plus faible pour le second (bleu plus intense). Jusqu'à $300^{\circ} \mathrm{C}$, la couleur initiale varie peu. Ensuite la couleur prend une teinte verte très pâle puis après chauffage à $600{ }^{\circ} \mathrm{C}$ puis $1000^{\circ} \mathrm{C}$ l'aérinite devient brune, la valeur de $L^{*}$ diminuant significativement (Tableau 2).

La mesure des coordonnées chromatiques permet de décrire objectivement ces modifications (Figure 3).

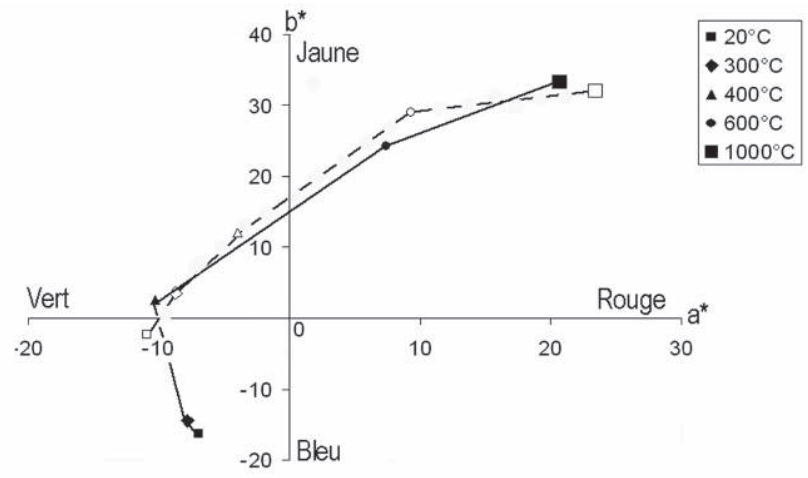

Figure 3 : Évolution des coordonnées chromatiques a* et $b^{*}$ de l'aérinite du Maroc (10360, en trait plein) et d'Espagne (10361, en pointillé) au cours du chauffage.

Figure 3: Evolution of colour coordinates $a^{*}$ and $b^{*}$ of aerinite from Morocco (10360, full line) and from Spain (10361, dotted line) during heating.

\begin{tabular}{|c|c|c|c|c|c|c|}
\hline \multirow{2}{*}{ Température $\left({ }^{\circ} \mathrm{C}\right)$} & \multicolumn{3}{|c|}{ Aeérinite du Maroc - 10360} & \multicolumn{3}{|c|}{ Aérinite d'Espagne - 10361} \\
\hline & $\mathrm{L}^{*}$ & $a^{*}$ & $b^{*}$ & $\mathrm{~L}^{*}$ & $a^{*}$ & $\mathrm{~b}^{*}$ \\
\hline 20 & 40,7 & $-7,0$ & $-16,3$ & 59,3 & $-10,9$ & $-2,3$ \\
\hline 300 & 40,2 & $-7,8$ & $-14,4$ & 59,5 & $-8,7$ & 3,5 \\
\hline 400 & 57,5 & $-10,3$ & 2,7 & 60,4 & $-4,0$ & 12,2 \\
\hline 600 & 58,6 & 7,4 & 24,3 & 63,9 & 9,3 & 29,0 \\
\hline 1000 & 49,7 & 20,7 & 33,2 & 41,3 & 23,4 & 32,0 \\
\hline
\end{tabular}

Tableau 2 : Coordonnées chromatiques L*a*b* mesurées pour des échantillons d'aérinite du Maroc et d'Espagne chauffés à différentes températures.

Table 2: $L^{*} a^{*} b^{*}$ colour coordinates measured for samples of aerinite from Morocco and Spain, heated at different temperatures. 
L'obtention de ces différentes nuances permet d'expliquer la variété des couleurs rencontrées dans les peintures murales romanes des Pyrénées et de leur altération lorsque ces peintures sont détruites par un incendie comme ce fut le cas à Santa-Maria de Sigena (Gasol Fargas, 1999) dont les peintures, déposées dans les années 1920, sont aujourd'hui conservées au Musée National d'Art Catalan à Barcelone.

Les diffractogrammes (Figure 4) établis sur l'aérinite de référence chauffée sont identiques jusqu'à $300^{\circ} \mathrm{C}$. La structure du minéral est conservée au moins jusqu’à cette température et change fondamentalement ensuite. Ils présentent les raies du quartz et celles de l'aérinite qui après chauffage à $1000^{\circ} \mathrm{C}$ ont disparu. La diffraction de rayons $\mathrm{X}$ montre que, comme dans les argiles calcaires, de nouvelles phases se sont formées à haute température, comme l'anorthite $\left(\mathrm{CaAl}_{2} \mathrm{Si}_{2} \mathrm{O}_{8}\right)$, résultant de la combinaison du $\mathrm{Ca}$ avec les aluminosilicates (Peters et Iberg, 1978). Parallèlement, le chauffage en atmosphère oxydante permet l'apparition de l'hématite $\left(\mathrm{Fe}_{2} \mathrm{O}_{3}\right)$, ce qui explique la couleur obtenue.
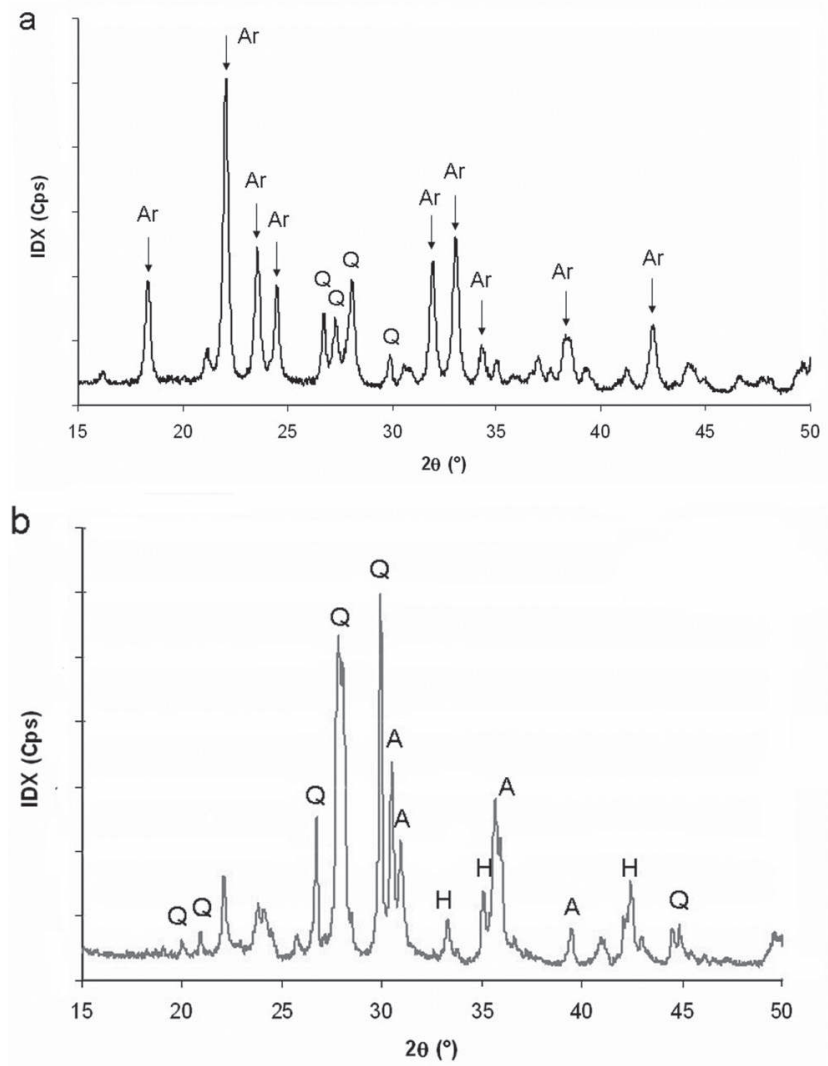

Figure 4: Diffractogrammes de l'aérinite avant (a) et après (b) chauffage à $1000^{\circ} \mathrm{C}$. (Ar : aérinite; Q : quartz; $\mathrm{A}$ : anorthite; $\mathrm{H}$ : hématite).

Figure 4: Diffractograms of aerinite before (a) and after heating at $1000{ }^{\circ} \mathrm{C}$. (Ar: aerinite; Q: quartz; A: anorthite; H: hematite).

\section{Identification des pigments bleus et verts prélevés sur les peintures murales de Moissac et de Nogaro}

\section{Pigments bleus et verts des peintures de l'église de Nogaro}

En ce qui concerne l'analyse des pigments des peintures murales de l'église de Nogaro, les échantillons ont été prélevés à des endroits différents sur la peinture (auréole de la tête du taureau, bande de la mandorle, à droite de la tête du Christ).

L'échantillon de pigment vert prélevé dans la tête du bœuf (10453) est identifié par spectrométrie Raman. La bande intense dédoublée à 335 et $365 \mathrm{~cm}^{-1}$ correspond à celle obtenue avecl'aérinite d'Espagne. Celles d'intensité plus faible à $515 \mathrm{~cm}^{-1}$ et $935 \mathrm{~cm}^{-1}$ correspondent également aux bandes Raman caractéristiques de l'aérinite (Figure 5) définies à l'aide de nos échantillons de référence.

L'analyse par MEB/EDS du pigment bleu dans la mandorle (10457) permet d'établir qu'il s'agit d'un pigment à base de cuivre, mais non de préciser sa nature. Le spectre Raman présente trois bandes : celles à $280 \mathrm{~cm}^{-1}$ et $1085 \mathrm{~cm}^{-1}$ sont caractéristiques de la calcite (le support de la fresque) tandis que celle à $532 \mathrm{~cm}^{-1}$ pourrait correspondre à la clinoatacamite (Bouchard et Smith, 2003), un chlorure basique de cuivre, produit de l'altération de l'azurite. L'azurite, carbonate basique de cuivre, $\mathrm{Cu}_{3}\left(\mathrm{CO}_{3}\right)_{2}(\mathrm{OH})_{2}$, bien que peu stable, est un des pigments bleus les plus utilisés dans le monde médiéval. Nous ne pouvons affirmer qu'il s'agit d'un pigment à base de cuivre et proposer l'hypothèse de l'emploi d'azurite, car les dimensions du micro prélèvement ne permettent pas l'identification par XRD qui aurait permis de lever l'ambiguité.

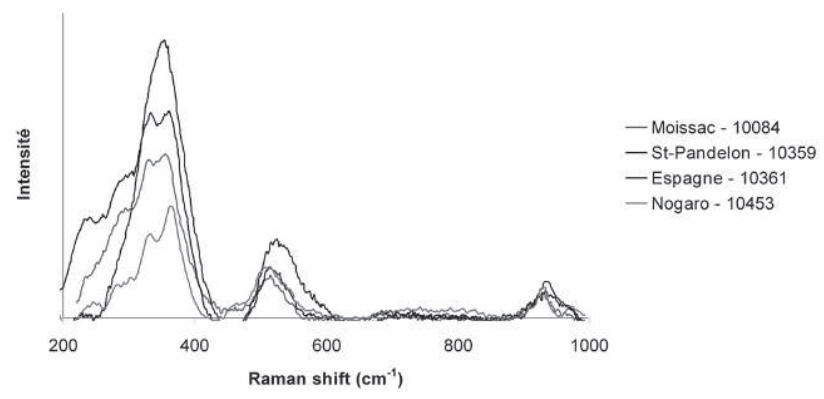

Figure 5 : Spectres Raman obtenus sur les pigments bleu (10084) de Moissac et vert (10453) de Nogaro ainsi que les spectres de l'aérinite de France (Saint-Pandelon, 10359) et d'Espagne (10361). Figure 5: Raman spectra on a blue pigment from Moissac (10084, a green pigment from Nogaro (10453), and reference aerinite from France (St-Pandelon, 10359) and Spain (10361). 
Quant au bleu à droite de la tête du Christ (10460), les analyses $\mathrm{MEB} / \mathrm{EDS}$ révèlent de fortes proportions de sodium, aluminium, silicium et soufre qui sont quatre éléments composant la lazurite $\left(\mathrm{Na}_{8-10} \mathrm{Al}_{6} \mathrm{O}_{24} \mathrm{~S}_{2-8}\right)$, un des constituants du lapis-lazuli (Plesters, 1966). La base de données Raman (Bell et al., 1997) mentionne pour le lapislazuli quatre bandes caractéristiques : $259 \mathrm{~cm}^{-1}, 546 \mathrm{~cm}^{-1}$, $805 \mathrm{~cm}^{-1}, 1093 \mathrm{~cm}^{-1}$ que nous retrouvons sur le spectre de ce prélèvement (Figure 6). C'est donc un pigment onéreux qui a été utilisé dans la partie centrale de la peinture, correspondant au Christ en majesté. Le coût du pigment obtenu à partir de cette pierre très dure était élevé en raison de sa provenance (Afghanistan), de la difficulté du travail d'extraction et des opérations de broyage et de purification. Bien que plus récente, une étude des comptes des ducs de Bourgogne à la fin du XIV siècle permet de comparer le prix des pigments à l'époque médiévale (Délivré, 2006). Malgré l'incertitude liée aux dénominations de pigments, il est possible d'estimer que le coût entre l'azur d'alemaigne (l'azurite était extraite des monts d'Allemagne et de Bohème) était 100 fois inférieur à celui de l'azur d'acre (lapis-lazuli). Quant à l'aérinite, nous n'avons pas d'indication, mais on pourrait supposer que, compte tenu de la disponibilité locale de la matière première, le coût devait être assez proche de celui des terres naturelles (ocres).

\section{Pigments bleus des peintures de la chapelle de Moissac}

La palette utilisée pour les peintures murales de la chapelle de l'ancien logis abbatial de Moissac est très riche et possède plusieurs sortes de bleus. L'analyse Raman a montré que le bleu foncé, employé pour le fond des mandorles, zone à forte valeur symbolique, est du lapis-lazuli. En ce qui concerne le bleu clair utilisé pour les fonds de la voûte de la chapelle, la microanalyse élémentaire a permis de détecter les éléments principaux d'une argile ( $\mathrm{Si}, \mathrm{Al}, \mathrm{Ca}, \mathrm{Fe})$. Sur les spectres Raman, nous retrouvons les bandes caractéristiques de l'aérinite. La figure 5 donne, à titre d'exemple, le spectre Raman obtenus sur un prélèvement de bleu ainsi que les spectres de l'aérinite d'Espagne et celle de St-Pandelon. Un seul échantillon provenant d'une zone proche d'un des symboles évangélistes (lion de saint Marc) présente, en plus de l'aérinite, quelques grains de lapis-lazuli et de noir de carbone. On peut supposer que l'ensemble de la voûte a été peinte avec de l'aérinite et que les rehauts blancs, les cernes noirs et les nuances de couleur ont été ajoutés ensuite. L'ensemble des résultats d'identification des pigments est donné dans le tableau 3.

Il semble donc que l'utilisation des bleus dans la partie romane ait été organisée en fonction de l'importance des thèmes iconographiques : le lapis-lazuli, pigment coûteux,

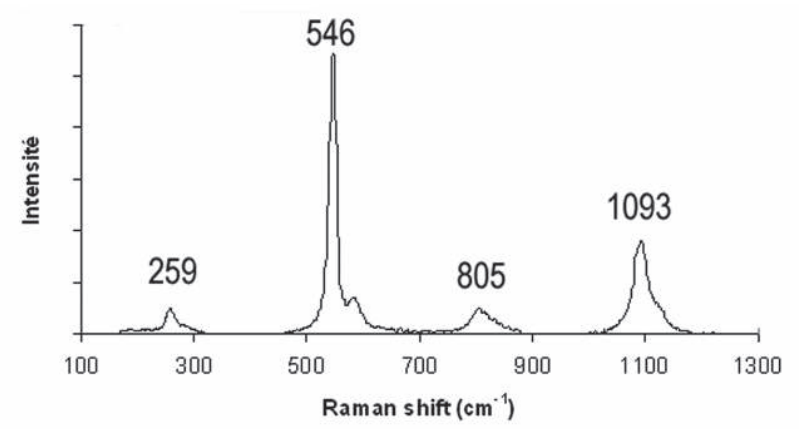

Figure 6 : Spectre Raman obtenu sur le pigment bleu (10460) de Nogaro. Les bandes correspondent à la lazurite.

Figure 6: Raman spectra on a blue pigment from Nogaro (10460). The bands correspond to those of lazurite.

est identifié à droite de la tête du Christ, dans la partie centrale de la peinture ou dans le fond; de l'azurite se trouve dans la mandorle, à l'extrémité de cette partie centrale, enfin, l'auréole dans laquelle s'inscrit le bœuf (représentation symbolique de saint Luc) ou bien l'ensemble des fonds de la voûte à Moissac est constituée d'aérinite, pigment bleu de valeur plus modeste et qui permet à moindre coût de colorer des surfaces plus étendues.

La palette utilisée pour les peintures est très riche et possède différents bleus : un bleu foncé pour le fond des mandorles des personnages principaux (David, la vierge en majesté, Christ en gloire) et un bleu clair couvrant le fond sur lequel est représenté le décor floral avec des entrelacs, un réseau de rinceaux gras et souples qui s'épanouissent en larges fleurs sur la voûte de la chapelle. Cette différence de tonalité permet de supposer que le choix de matériaux a été influencé par l'iconographie.

D'un point de vue stylistique, les peintures murales de Moissac ont été rapprochées de celles de la salle capitulaire du monastère Santa Maria de Sigena en Aragon (XII siècle) : une même iconographie (l'arbre de Jessé), des similitudes dans le répertoire décoratif comme, par exemple, l'utilisation de l'octopus flower, issu du répertoire anglo-normand (Czerniak, 2004).

\section{ConClusion}

Peu valorisé auparavant dans l'occident médiéval, le bleu reste encore une couleur discrète avant le $\mathrm{XII}^{\mathrm{e}}$ siècle, mais son utilisation, associée à l'image de la Vierge, ne va cesser de s'accroître pour jouer un rôle essentiel. "Pour plusieurs siècles, il y aura dans l'art occidental presque synonymie 


\begin{tabular}{|l|l|l|}
\cline { 2 - 3 } \multicolumn{4}{c|}{} & Réf. éch. & Pigment identifié \\
\hline \multirow{4}{*}{ Chapelle de l'ancien logis abbatial de Moissac } & 10073 & lazurite + aérinite \\
\cline { 2 - 3 } & 10083 & aérinite \\
\cline { 2 - 3 } Saint-Nicolas de Nogaro & 10084 & aérinite \\
\hline & 10365 & aérinite \\
\cline { 2 - 3 } & 10453 & aérinite \\
\cline { 2 - 3 } & 10457 & ? Pigment à base de cuivre \\
\hline & 10460 & lazurite \\
\hline
\end{tabular}

Tableau 3 : Identification des pigments prélevés dans les peintures murales de Moissac et de Nogaro.

Table 3: Results of the identification of the pigments from mural paintings of Moissac and Nogaro.

entre la lumière, l'or et le bleu " (Pastoureau, 2002). Pour les bleus, on utilise dans les peintures murales, à cette époque, du lapis-lazuli pour le bleu vif, localisé dans les zones importantes (Christ, Vierge), de l'azurite et, cas particulier du nord de l'Espagne, de l'aérinite. A Moissac, l'aérinite a été employée pour réaliser le fond des scènes, des entrelacs floraux. L'emploi du lapis-lazuli est réservé aux scènes symboliquement importantes (Christ, Vierge en Majesté). Par économie, notamment, il peut être appliqué sur une couche d'aérinite ou de noir de carbone.

L'étude expérimentale menée sur des échantillons géologiques d'aérinite montre que de nombreuses nuances de couleur peuvent être obtenues par chauffage (volontairement ou involontairement, comme dans le cas des incendies). Des études plus approfondies de l'effet de la déshydratation sur les transformations de phase de l'aérinite et leur suivi par spectrométrie Raman et par diffraction X, une comparaison des différents gisements et, à l'intérieur d'une même source, de la variabilité de ses caractéristiques physico-chimiques et, enfin, de l'influence de la granulométrie sur la perception colorée et permettrait une plus juste connaissance de l'emploi de ce pigment et de son identification, même s'il a subi des altérations, de l'origine de la matière première et de sa mise en œuvre par les artistes de l'époque romane.

La découverte de ce pigment, aux nuances bleues ou vertes, dans la chapelle de l'ancien logis abbatial de Moissac (XII ${ }^{\mathrm{e}}$ siècle, Tarn et Garonne) et à l'église Saint-Nicolas de Nogaro (fin $\mathrm{XI}^{\mathrm{e}}$ siècle, Gers) ainsi que les relations iconographiques et stylistiques qui ont été établies précédemment entre les sites français (Moissac) et certains sites espagnols (Sigena) sont un indice des échanges transpyrénéens (matériaux, ateliers) à l'époque romane.

\section{Remerciements}

Cette étude a été réalisée dans le cadre d'un projet soutenu par la Communauté de Travail des Pyrénées.
Nous remercions Jean-Marc Stouffs et Didier Legrand, conservateurs-restaurateurs, ainsi que Chantal Fraïsse, conservateur au Centre d'Art Roman de Moissac et Virginie Czerniak, Maître de conférences d'histoire de l'art médiéval à l’Université de Toulouse Le Mirail.

\section{Bibliographie}

Azambre, B. et Monchoux, P., 1988. Précisions minéralogiques sur l'aérinite : nouvelle occurrence à Saint-Pandelon (Landes, France), Bulletin Minéralogique, 111, p. 39-47.

Bell, I. M., Clark, R. J. H. et Gibbs, P. J., 1997. Raman spectroscopic library of natural and synthetic pigments (Pre- $\sim 1850$ AD), Spectrochimica Acta Part A, 53, (2), p. 2159-2179.

Bouchard, M. et SMith, D. C., 2003. Catalogue of 45 Raman spectra of minerals concerning research in art history or archaeology, especially on corroded metals and coloured glass, Spectrochimica Acta, Part A 59, p. 2247-2266.

Coupry, C., 1991. Apports et limites de l'analyse des pigments colorés par la méthode de spectroscopie Raman. Enduits et mortiers : archéologie médiévale et moderne, Paris, CNRS.

CzerniaK, V., 2003. Les peintures murales de la chapelle de l'ancien logis abbatial de Moissac. Un exemple méridional de l'influence des Plantagenêt?, Mémoires de la Société Archéologique du Midi de la France, LXIII.

Czerniak, V., 2004. La peinture murale médiévale en Quercy: $X I I^{e}-X V I^{e}$ siècle, Thèse de doctorat d'Histoire de l'Art Médiéval, Université de Bordeaux 3, p. 88-96.

Délivré, J., 2006. Les comptes des ducs de Bourgogne à la fin du XIV ${ }^{\mathrm{e}}$ siècle. Les noms et les prix des pigments, $12^{\text {es }}$ journées d'études de la SFIIC, Couleur et temps. La couleur en conservation et restauration, Paris, 22-23 juin 2006, p. 132-142.

Downs, R. T., 2006. The RRUFF Project : an integrated study of the chemistry, crystallography, Raman and infrared spectroscopy of minerals. Program and Abstracts of the 19th General Meeting 
of the International Mineralogical Association in Kobe, Japan, $\varnothing 03-13$.

El Agrchmi, M., 1981. Etude de la déshydratation de l'aérinite, Rapport de DEA, Université de Toulouse.

Gasol Fargas, R. M., 1999. Study of the original technique of the wall painting of the chapter house of Santa Maria de Sigena, Spain (1190-1200), 12 th ICOM triennial meeting, Lyon, 29 août-3 septembre 1999, volume II, p. 467-472.

Palet Casas, A. et de Andres Llopis, J., 1992. The identification of aerinite as a blue pigment in the romanesque frescoes of the pyrenean region, Studies in Conservation, 37, p. 132-136
Pastoureau, M., 2002. Bleu. Histoire d'une couleur, Paris, Seuil, $219 \mathrm{p}$.

Peters, T. et Iberg, R, 1978. Mineralogical changes during firing of Calcium-Rich Brick Clays, American Ceramic Society Bulletin, Vol. 57, n 5, p. 503-509.

Plesters, J., 1966. Ultramarine Blue, Natural and Artificial, Studies in Conservation, 11, (2), p. 62-91.

Porta, E., Palet, A. et Guilamet, E., 1990. Le bleu aérinite : un pigment méconnu en peinture murale romane, $X^{e}$ réunion triennale de l'ICOM-CC, vol. 2, p. 534-538. 